\title{
Wind and Photovoltaic Power Ratio Model in Hybrid Wind and Photovoltaic Power Module Applied to Online Monitoring System for Transmission Line
}

\author{
Zeyu Cui
}

School of Electrical and Electronic Engineering Department, North China Electric Power University, Baoding 071003, China

cuizeyu15@qq.com

Keywords: Wind and Photovoltaic Power Ratio Model, optimization model, proportion.

\begin{abstract}
To analyses the proportion of wind and solar in a hybrid wind and photovoltaic power systems in the online monitoring system for transmission line, several experiments are made in order to get the environment data. To build an optimization model, a marketing research is set out, and the relation between the equipment type and the cost is calculated. By the optimization model, the requirement of detection system is taken into consideration, and the best proportion could be set.
\end{abstract}

\section{Introduction}

Monitoring systems are generally erected on top of the tower and get power from solar panels, wind turbine or CT-based coils. Solar panels and small wind turbines both depends on local weather conditions, which makes the output voltage unstable. In order to extend battery service time, hybrid wind and photovoltaic power systems often go through a power conversion module.

We conduct experiments to analyze a good system energy ratio. Our experiment is based on the premises that wind and solar timing problem has been well solved through controller.

\section{Hybrid Wind and Photovoltaic Power System}

A basic hybrid wind and photovoltaic power system is mainly composed of a control module, small wind turbines, solar panels, batteries, several parts. In this paper the complementary is considered to be done by the control modules, which won't be mentioned in the following discussion.

Wind Turbine Energy Conversion Formula. The wind generator is the wind turbine mechanical energy into electrical energy device.

The energy conversionformula [1] are:

$$
P_{W}=\left\{\begin{array}{lr}
0 & V<V_{\min } \\
\frac{1}{2} C_{p} \pi \rho R^{2} V^{3} & V_{\min }<V<V_{c} \\
P_{c} & V_{c}<V<V_{\max } \\
0 & V_{\max }<V
\end{array}\right.
$$

Where $P$ — the output powerW;

$C_{p}$ — the power coefficient which is used to be $50 \%[2]$;

$\rho$ - the density of air $\mathrm{kg} / \mathrm{m}^{3}$;

$V$ — wind speed $m / s$;

$R$ — the wind wheel radiusm.

Photovoltaic energy conversion formula. Photovoltaic cells convert solar energy directly to electrical energy device, its Energy conversion formula [3] are;

$E=H \times \eta \times K_{P} \times K_{S} \times A / 3.6(2)$

Where, $E$ - output powerw $/ \mathrm{m}^{2}$;

$H_{i} \longrightarrow$ unit area radiationw $/ \mathrm{m}^{2}$;

$\eta-$ conversion efficiency, could be $8 \%$;

$K_{P} \longrightarrow$ package factor coefficient, used to be 0.9 ;

$K_{S}$ — Dust accumulation coefficient, used to be 0.96; 
$A —$ the area of the photovoltaic cellm ${ }^{2}$

\section{Wind and Photovoltaic Power Ratio Model and Results in Baoding}

Power Analysis on Wind Generator.We measure wind speed from 3:30 pm to 6:30 pm and from 7:30 pm to 9:30 pm every day. The data is shown in Fig.1.

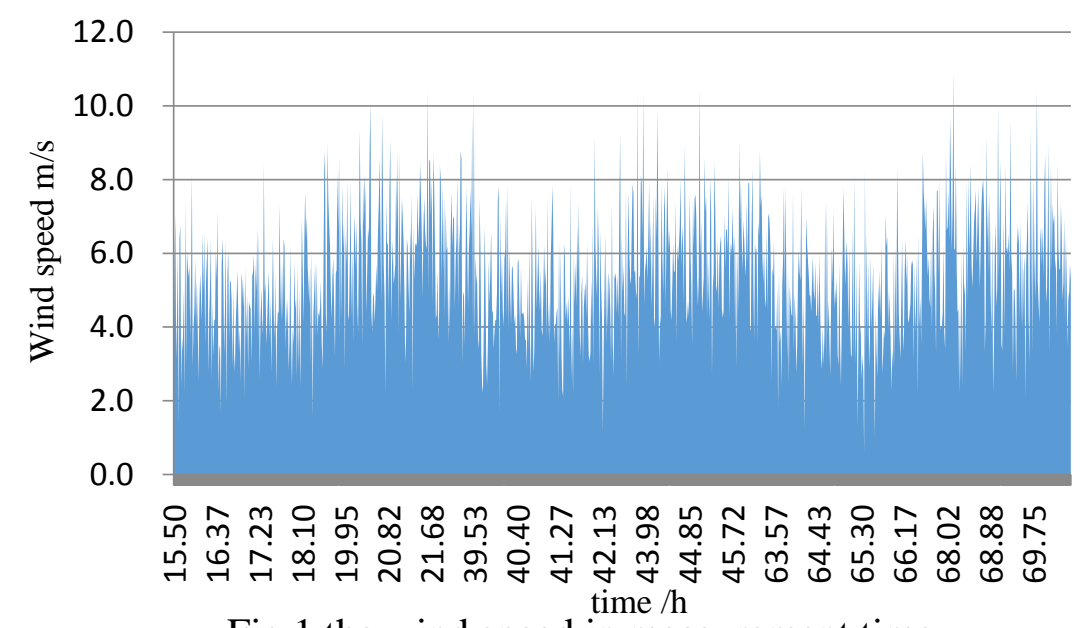

Fig. 1 the wind speed in measurement time

We put the data above into equation (1). Wind energy utilization is $50 \%$ and air density can be assumed as standard condition.

$$
\begin{aligned}
& W=\int_{0}^{t} \frac{1}{2} C_{p} \rho \pi R^{2} v^{3} \mathrm{dt}(3) \\
& W=R^{2} \times \int_{0}^{t} \frac{1}{2} C_{p} \rho \pi v^{3} \mathrm{dt}(4) \\
& W=R^{2} \times \mathrm{S}(5)
\end{aligned}
$$

From equations above, we can estimate that;

$P=s \times R^{2}(6)$

Through Mat lab, we calculate s value at each time (shown in Fig. 2).

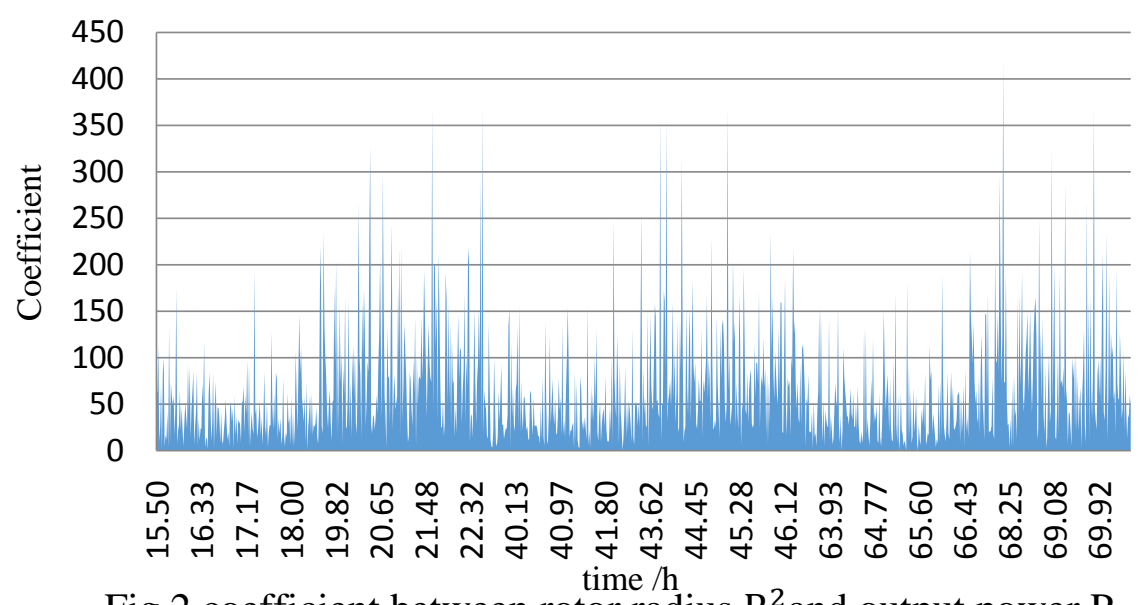

Fig. 2 coefficient between rotor radius $\mathrm{R}^{2}$ and output power $\mathrm{P}$

Thus, the relationship between the average output power and the radius is;

$P=65.4071 \times R^{2}(7)$

Where coefficient is from the calculation of the wind speed in three days in Baoding.

Power Analysis on Photovoltaic Panel. We measureillumination from 8:30 am to 11:30 am and from 3:30 pm to 6:30 pm. Three days' illumination is in Fig.3. To reflect its trend more clearly, we use logarithmic to express. 


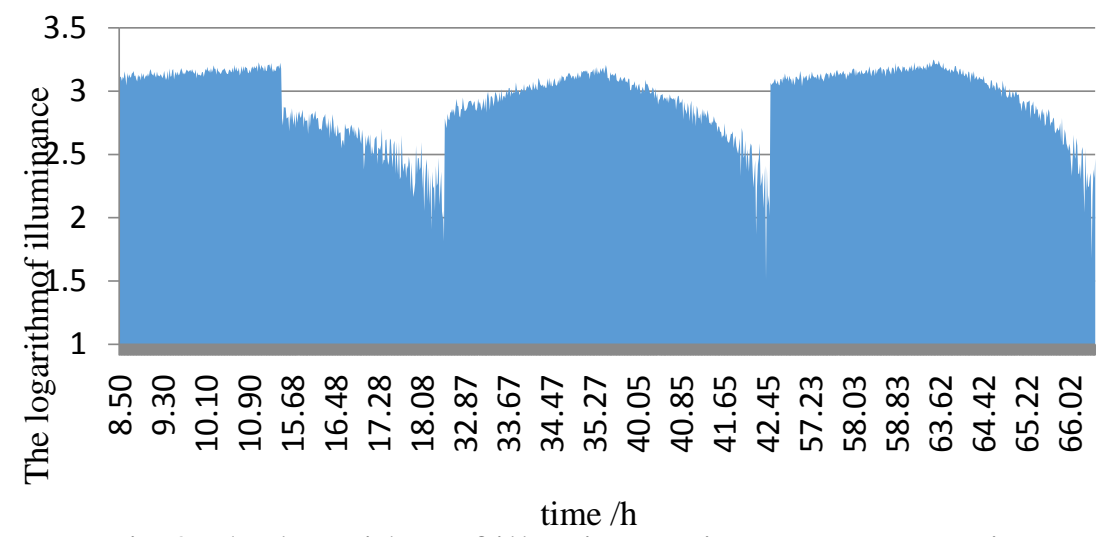

Fig. 3 The logarithm of illuminance in measurement time According to paper [4], the conversion formula is: $E=1.04 \times 10^{-2} L(8)$

After calculation, the radiation degree per unit area is shown in Fig. 4.

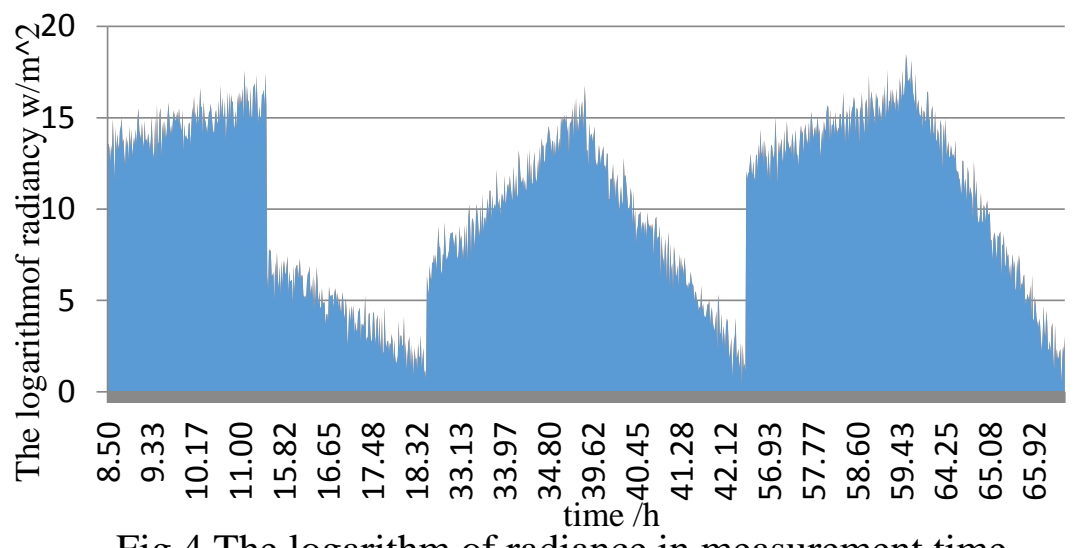

Fig.4 The logarithm of radiance in measurement time

Thus, the average amount of radiation per hour is $12.01924 \mathrm{~W} \cdot \mathrm{m}^{2}$. Then we put it into equation (2).

$E=12.01924 \times 0.08 \times 0.9 \times 0.96 \div 3.6 \times A(9)$

And get equals to0.230769A.

\section{Cost Analysis on Wind Generator and Photovoltaic Power}

Table. 1 The market price of different rotor diameter, rated power and price

\begin{tabular}{c|c|c}
\hline Rated power & rotor diameter & price \\
\hline 100.00 & 0.82 & 520.00 \\
200.00 & 1.10 & 680.00 \\
300.00 & 1.30 & 1086.00 \\
400.00 & 1.55 & 1280.00 \\
600.00 & 1.70 & 1680.00 \\
1000.00 & 1.96 & 1980.00 \\
2000.00 & 3.20 & 7950.00 \\
3000.00 & 3.70 & 11340.00 \\
5000.00 & 6.20 & 25600.00 \\
10000.00 & 8.00 & 30290.00 \\
\hline
\end{tabular}




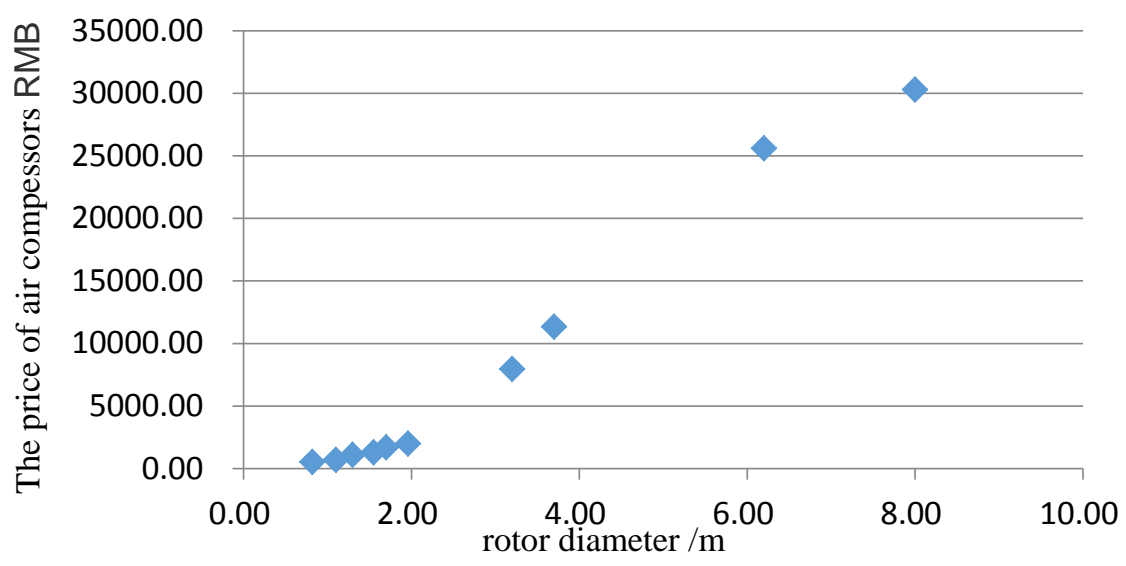

Fig. 5 the relation between rotor diameter and price

Analysis of Wind Power Generator Cost. By curve fitting, a formula could be set: $y=-169.69 D^{3}+2244 D^{2}-3575 D+2075(10)$

Analysis of Photovoltaic energy cost. The general price of solar panel is 460 yuan per square meters, so the cost of Photovoltaic energy cost is:

$y=460 A(11)$

\section{The Optimization Model of the Proportion of Wind and Solar}

As is discussed above, an optimization model could be formed:

$M$ in $=460 \mathrm{~A}-169.69 \mathrm{D}^{3}+2244 \mathrm{D}^{2}-3575 \mathrm{D}+2075(12)$

The online monitoring system for transmission line usuallyconsumes $5 \mathrm{w}$ loads, take three times allowance into consideration, $15 \mathrm{~W}$ is the final loads. Thus the constraint equation are:

$P=65.4071 \times(D / 2)^{2}+0.230769 A=15(13)$

So the final optimization model is:

$$
\operatorname{Min}=
$$

$$
460 A-169.69 D^{3}+2244 D^{2}-3575 D+2075\left\{\begin{array}{c}
A>0 \\
D>0 \\
65.4071 \times(D / 2)^{2}+0.230769 A=15
\end{array}\right.
$$

The solution is:

$D=0.999 A=0.950$

That is the best proportion is to use $0.999 \mathrm{~m}$. The wind wheel radius and the size of solar panel shall be $0.95 \mathrm{~m}^{2}$.

\section{References}

[1] Z.L. Sun, Study on the Efficiency Problem of the Solar Battery, (2009) No.9, p.34.

[2] C.H. Hu and K.H. Wang, Study on the Optimal Ratio of the Hybrid Wind and PV Power System in North Xinjiang, Vol.364 (2013) No.1, p.69.

[3] H.Y. Li, L.X. Liu, S.S. Niu and L. Zhao, Energy Match Characteristics Study of Stand Alone Hybrid Wind and PV Power System, Vol.166(2011) No.10, p.36.

[4] J.X. Mao, Solar Irradiance and Illuminance Conversion Analysis,(1995) No.1, p.11. 